\title{
Consecutive Primes in Arithmetic Progression
}

\section{By L. J. Lander and T. R. Parkin}

A. Schinzel and W. Sierpiński [1] conjectured that there exist arbitrary long arithmetic progressions formed of consecutive prime numbers. Sierpiński stated in [2] that a progression of five consecutive primes had not yet been found. A direct computer search showed that the first such progression has the common difference $d=30$ and begins with the prime $9,843,019$. The first progression of six consecutive primes begins with $121,174,811$ and also has $d=30$. Up to the limit $3 \times 10^{8}$ there are 25 other progressions of five consecutive primes, all with $d=30$; there are no other progressions of six consecutive primes.

The referee points out that recently a much larger quintuplet, beginning with 10000024493, and again having $d=30$, was recorded [3], but without reference to Sierpiński's remark. The smaller set that we found, and the single sextuplet, may still be worth recording.

Aerospace Corporation

Los Angeles, California 90045

1. A. Schinzel \& W. Sienpióski, "Sur certaines hypothèses concernant les nombres premiers," Acta Arith., v. 4, 1958, p. 191. MR 21 \#4936.

2. W. SienPiński, A Selection of Problems in the Theory of Numbers, Macmillan, New York, 1964, p. 105. MR 30 \#1078.

3. M. F. Jones, M. LaL \& W. J. Blundon, "Statistics on certain large primes," Math. Comp., v. 21, 1967, pp. 103-107.

Received November 28, 1966.

\section{Convergence of Successive Substitution Starting Procedures}

\section{By A. C. R. Newbery}

The method of successive substitutions (also known as Picard's method) has been proposed [1], [2] as a means of initialising the numerical solution of the differential equation $x^{\prime}=f(x, t)$. The method is capable of advancing the solution $k$ steps at an average cost of $k$ function-evaluations per step with a truncation error of order $O\left(h^{k+2}\right)$. This makes it potentially one of the most efficient methods available for the purpose, and so it seems appropriate to study its numerical convergence properties. The method is based on $k$ formulas of the form $x_{r}=x_{0}+$ $h L_{r}\left(x_{0}{ }^{\prime}, x^{\prime}, \cdots, x_{k}{ }^{\prime}\right), r=1,2, \cdots, k$ where, $L_{r}$ denotes a linear combination with known constant coefficients. The required coefficients are implicit in the corrector matrices published in [3]. For a given $k$, the coefficients in $L_{r}$ are the entries in the $r$ th column of the $k$ th corrector matrix. For example with $k=2$ we would obtain the formulas:

$$
x_{1}=x_{0}+(h / 24)\left(10 x_{0}{ }^{\prime}+16 x_{1}{ }^{\prime}-2 x_{2}{ }^{\prime}\right), x_{2}=x_{0}+(h / 24)\left(8 x_{0}{ }^{\prime}+32 x_{1}{ }^{\prime}+8 x_{2}{ }^{\prime}\right) .
$$

